\title{
AUTOMATED SHAPE ANALYSIS OF TEETH FROM THE ARCHAELOGICAL SITE OF NERQIN NAVER
}

\author{
A. Gaboutchian ${ }^{a^{*}}$, H. Simonyan ${ }^{\text {b }}$, V. Knyaz ${ }^{\text {c,d }}$, G. Petrosyan ${ }^{\text {e }}$, L. Ter-Vardanyan ${ }^{\text {e }}$ N.A. Leybova ${ }^{\text {f }}$, S.V. Apresyan ${ }^{\mathrm{g}}$ \\ ${ }^{a}$ Moscow State Medical-Stomatological University, Moscow, Russia - armengaboutchian@mail.ru \\ ${ }^{\mathrm{b}}$ Scientific Research Centre of the Historical and Cultural Heritage, Yerevan, Armenia - haksimon@ gmail.com \\ ${ }^{c}$ State Research Institute of Aviation System (GosNIIAS), Moscow, Russia - knyaz@ gosniias.ru \\ ${ }^{\mathrm{d}}$ Moscow Institute of Physics and Technology (MIPT), Russia \\ ${ }^{\mathrm{e}}$ International Scientific-Educational Center of NAS RA, Yerevan, Armenia - petrosyan_gohar@list.ru \\ ${ }^{\mathrm{f}}$ Institute of Ethnology and Anthropology of Russian Academy of Sciences , 119991, Moscow, Russia - nsuvorova@mail.ru \\ g Peoples’ Friendship University of Russia, 117198, Moscow, Russia - dr.apresyan@ gmail.com
}

Commission II, WG II/10

KEY WORDS: Automated Digital Odontometry, Photogrammetry, Tooth shape analysis, Odontometry, Odontotomy, Nerqin Naver

\begin{abstract}
:
Traditional odontometry currently suggests a limited number of measurements on tooth coronal parts, including estimation of mesiodistal and vestibular-oral diameters, or dimension, through usually a single measurement of the maximal parameter. Taking into consideration the complexity, irregularity and variability of tooth shapes we find such measurements insufficient for interpreting tooth morphology. Thus we propose odontotomic approach of obtaining data from a series of parallel equally spaced sections in combination with automated detection of landmarks used for measurements. These sections allow locating maximal dimensions of teeth as well as collecting data from all parts of the tooth to describe it morphologically. Referring odontometric data to the whole tooth we obtain more precise and objective records which have proved to be informative in a series of dental and anthropological studies.
\end{abstract}

Growing interest and implementing of digital technology in odontometric studies calls for studies ensuring transition to new methods. The current research is aimed to undertake a comparative study of the traditional and automated digital odontometry. The influence of various approaches to odontotomy (number and direction of sections) on odontometric data is subjected to studies as well. The above-mentioned tooth shape analysis is applied to samples from the archaeological site of Nerqin Naver to contribute to complicated odontological studies from the Early Bronze burials.

\section{INTRODUCTION}

\subsection{Odontometric Techniques}

Tooth morphology studies include various research methods, among which traditionally measuring methods have been in the background of methods called descriptive. "Dental morphologists focus on structural details of tooth crowns and roots and pay less heed to other methods of characterizing tooth shape" (Scott and Turner, 1997). Reasons seem to be in the limitations of widely used measurement methods, since they mainly represent estimation of maximal sizes of crowns or their parts in two dimensions (Peiris et al., 2013).

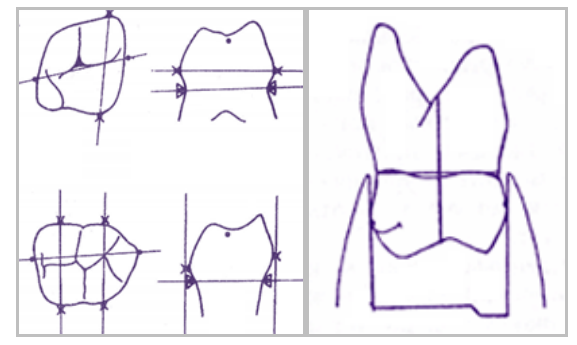

Figure 1. Guidelines for measurements on tooth crowns.
Providing important data on sizes of teeth and dental arches (Hasegawa et al., 2014) on one hand such measurements are insufficient for reflecting morphological complexities of teeth on the other (Figure 1, from A.A. Zubov, 1968 and S.V. Dmitrienko et al., 1998).

Odontometry, being an integrated part of anthropological studies, has a history of gradual development. Despite the apparent simplicity of measurements, including two basic mutually perpendicular dimensions of tooth crown and cervix (MD - mesio-distal and VL - vestibulo-lingual), crown height and root length, a number of different techniques exist. Small sizes of teeth, variety of measurement techniques and uncertainty of the obtained results have even generated discussions on excepting of odontometry from studying methods. Nevertheless, odontometry have repeatedly evidenced to be informative in anthropological studies, and the search of ways in measurements' improvement continues today.

Traditionally contact measurements of teeth are conducted by means of modified caliper with sharpened edges for use on dental arches, when studied teeth cannot be removed from alveolar sockets. Tooth crown measurements in mesio-distal and vestibular-lingual directions are considered to be the most relevant. Researchers meet difficulties predominantly while

\footnotetext{
* Corresponding author
} 
measuring molars, especially upper. According to the method suggested by R. Martin, MD-diameter is estimated empirically as maximal; maximal VL-diameter is perpendicular to MD and the caliper is positioned vertically along the longitudinal tooth axis. This measurement method has been widely implemented in odontometric studies (Irish and Scott, 2016) serving for obtaining wide-ranging data on sizes of molars. A number of attempts to improve odontometry have been made in recent decades, among such - measurements of inter-cuspal distances (Sekikawa et al., 1988), tooth crown diagonal measurements (Hillson et al., 2005) and others.

The traditional and similar to it methods have been applied during studies to different objects: separate teeth and dental arches, their copies and images, including photogrammetrically obtained (Horton et al., 2010; Fleming et al., 2011; Paredes et al., 2015; Townsend et al., 2012). Replication of well-known methods on digital images certainly has its exploratory importance (Figure 2, Zilberman et al., 2003), but does not really disclose that benefits which up-to-date digital technology can bring to odontology.
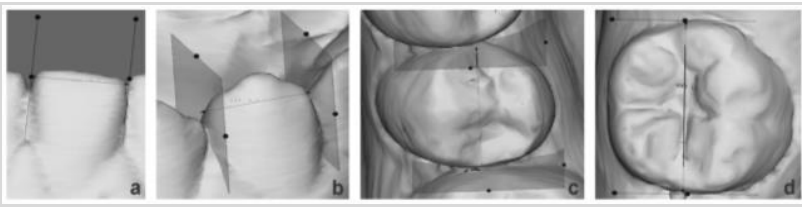

Figure 2. Measurements of teeth on 3D images

In turn, we suggest for crown measurements automated digital odontometry based on methods earlier applied to a number of studies in dentistry and anthropology (Gaboutchian et al., 2017 a, b). Together with inherent to measurement-based methods objectivity, automated digital odontometry allows obtaining of variable, previously inaccessible data within a short period of time and with high accuracy.

Currently the measurements are conducted on odontotomic contours, obtained through sectioning 3D images by parallel equally spaced planes running through entire studied area of teeth. Presently the odontometric software allows up to 100 section odontotomy and further measurements in an automated mode.

The increase in the number of studied sections is not the only factor providing for study objectivity. The applied methods allow obtaining of up to about two hundred odontometric parameters on each section (Figure 3), certainly including the ones which are measured traditionally: maximal mesio-distal and vestibular-oral dimensions of tooth crowns. Thus similar odontometric parameters obtained by different methods are subjected to study and presented in this paper.

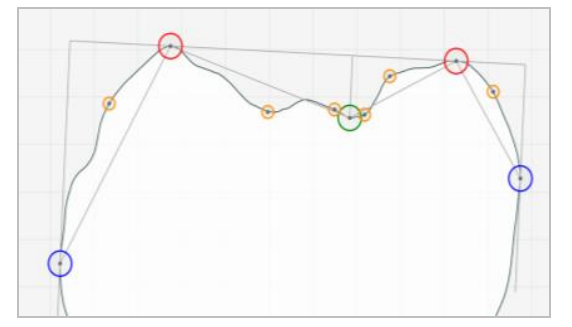

Figure 3. Automatically set landmarks on a tooth section

\subsection{Archaeological Background}

The archaeological site of Nerqin Naver is located on lands of Parbi village, Aragatzotn region, Republic of Armenia, on the left bank of Shaghvert River. An extensive burial complex covers an area of 40 hectares. Explorations of Nerqin Naver kurgans undertook in 2002-2015 during expeditions held by the Scientific Research Centre of Historical and Cultural Heritage of Ministry of Culture, RA (Figure 4). 11 kurgans have been excavated over these years and some of them, among various archaeological artefacts, contain only teeth and phalange bones as human remains. Thus in Burial Nr 3 (Figure 5) 114 human teeth of various classes, conditions and from different individuals were found (according to preliminary estimates they can belong to at least $14-17$ individuals). For this reason odontological analysis is important element in the studies of Nerqin Naver.

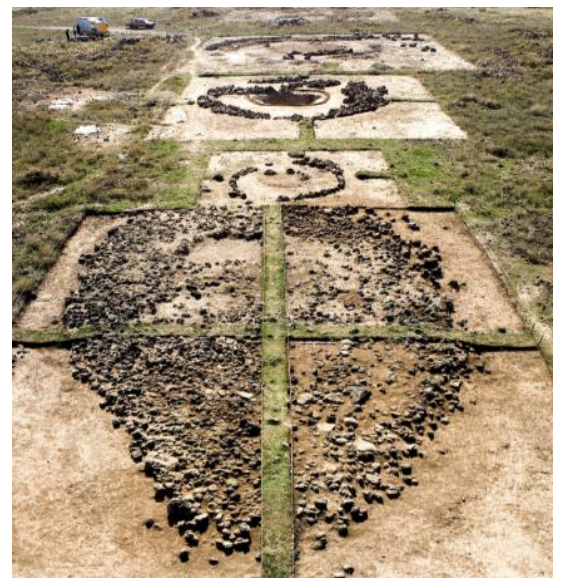

Figure 4. Archaeological fields of Nerqin Naver

The mentioned archaeological findings reveal accordance to proto-zoroastric burial rites: the body of dominus was cremated; the bodies of sacrificed concubines and servants were placed in specially designated places - Towers of Silence or Dakhmas, where their bodies were exposed to carrion birds. Thereafter the skeletal remains were buried together with cremated remains of the dominus. Archaeological studies demonstrate wide dissemination of Indo-European mythology and worldview among Bronze Age aristocracy in Armenia.

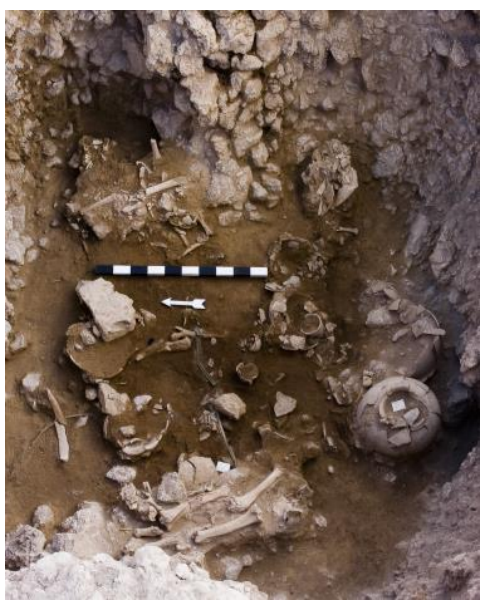

Figure 5. Burial $\mathrm{Nr} 3$ of Nerqin Naver 
The number of individuals buried in kurgans, their social standing and distribution by gender, age, ancestral ties and ethnic origin are questions of interest from the archaeological point of view. Odontological findings from Nerqin Naver burials represent a complicated fragmented material while remaining an essential source of information. As we believe, precise non-contact odontometric methods and morphometric analysis of teeth have the potential in revealing a number of archaeological and historical nuances referring to the burials of the Armenian Highlands inhabitants in the XXIV-XVIII centuries BC. Odontological studies of findings from Nerqin Naver burial complex can complement results obtained through implementation of other methods, such as DNA analysis, giving an impetus for new historical and anthropological interpretations.

\subsection{Application of Automated Digital Odontometry}

As mentioned before, automated digital odontometry allows obtaining variable data referring to shape of tooth coronal parts. Parameters describe linear dimensions, angles, contour lengths, areas and volumes. Measurements can be applied to the whole crown of its different parts: vestibular or oral cusps, inner of outer cusp slopes or anatomic occlusal surface. Depending on the objectives absolute figures, their ratios or complex coefficients are studied and analysed. Two main spheres of automated digital odontometry application are anthropological and dental studies.

1.3.1. Applications in Anthropology: Linear measurements traditionally conducted by calipers, or odontometers, have been the only source of metric data for paleoanthropologists for decades. Instrumentation capabilities, variability of odontological samples (separate teeth, complete or partial dental arches, teeth subjected to erosion and attrition, chipped and cracked dental tissues), variability of crown shapes within and between different classes of teeth and limitations of existing measurement techniques in comparison to morphological complexity of teeth have considerably influenced on implementation of measurements in anthropology. At the same time multiple original descriptive approaches have been developed and used in odontological studies. However, appropriate training and experience is required for correct estimation of odontological features and, especially, of their expression level.

Automated digital odontometry and 3D shape analysis has a potential to enrich research methods in anthropology. Thus a preliminary study of cusp slopes' inclination measurements presented at PSBB workshop in May 2017 (Moscow) has found its development. Results of a more profound comparative odontometric study dealing with angulations of coronal surfaces on samples from various historical periods will be presented in the near future.

1.3.2. Applications in Dentistry. Computer aided technologies based on 3D image obtaining and processing are widely used in dentistry today. In line with traditional manual techniques, computer aided manufacturing and 3D printing have taken hold in fabrication of various therapeutic, supplementary and restorative constructions. Another direction where computer technologies are used in dentistry is treatment planning regarding proper aesthetic and functional reconstruction, surgical procedures, orthodontic treatment, etc. Thus shape analysis, being applied to teeth, dental arches and soft tissues, contributes to predictability of treatment results and understanding between dentist and patient by the use of smile design techniques which are in demand in aesthetic dentistry.

In turn, having a positive experience of digital odontometry and occlusiometry application in the analysis of tooth crown shapes and of interrelations between opposing teeth, we put forward studies based on automated measurements. Together with research presented in this article we are in progress with a study for dental applications as well. Shapes of artificially designed tooth crowns fabricated on various computer-aided appliances are compared between each other and with manually fabricated ones by means of the developed odontometric methods. The study is aimed to reveal pre-set capabilities of dental prosthesis design software and impact of dental technicians' interpretation of tooth shape on the final clinical results (both, functional and aesthetic).

\section{STUDY MATERIALS AND TECHNIQUES}

\subsection{Material}

The study has been carried out on teeth from burials 1 and 3 (around XXII century BC) of Nerqin Naver. In total 138 teeth of various shapes, types, conditions and from different individuals were scanned. 9 molars and 1 premolar from the both burials were picked for this study. The teeth were measured manually, their 3D images were measured digitally in an automated mode.

\subsection{D image generation}

The teeth were scanned using photogrammetric methods and facilities. Images were obtained partly on professional dental equipment - Zfx Evolution plus (Figure 6) - with scan accuracy $<9 \mu \mathrm{m}$; the other part - on a photogrammetric scanning system originally designed at GosNIIAS, providing $10 \mu \mathrm{m}$ accuracy. Both approaches have reasonable accuracy and, therefore, the obtained 3D images are applicable for studies.

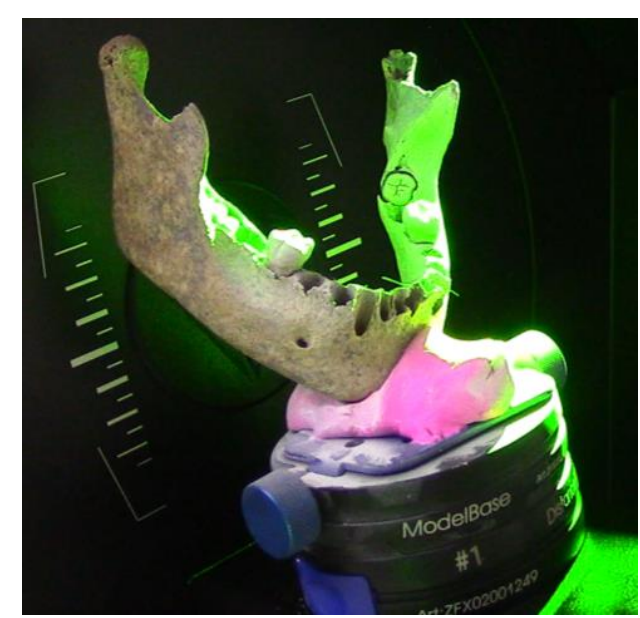

Figure 6. Scanning of palaeoanthropological samples

\subsection{Software}

The digital part of measurements was conducted by means of appropriate software. Two separate versions: one for automated digital odontometry, another - for imitation of manual measurements on digital images were developed. Among general features providing operability of 3D images, the 
software possesses specific functions for odontometric applications, such as import of images in various formats, automated odontotomy, automated measurements on plane contours and 3D models, data acquisition and calculation of odontometric parameters, and many others allowing adjustments during measurements.

\subsection{Odontometric Methods}

Measurement data used in the study for analysis and comparisons was obtained by application of two methods: manual odontometry and automated digital odontometry.

2.4.1. Manual Odontometry: Measurements were conducted on 10 teeth by means of digital caliper (odontometer) with accuracy of $0.1 \mathrm{~mm}$. Methods accepted in anthropology for odontometric studies were applied during measurements (Figure 7). Two maximal parameters were estimated on each tooth crown: mesio-distal and vestibular-oral diameters.

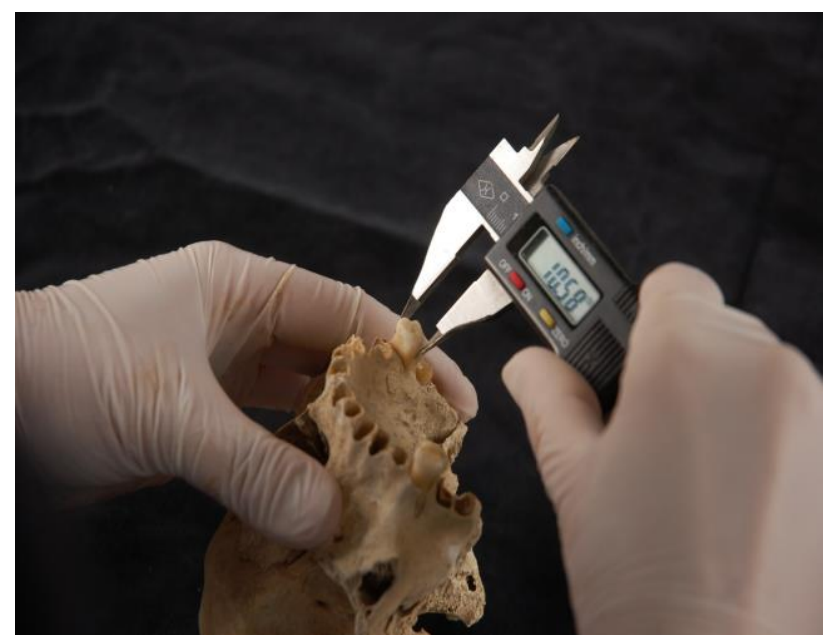

Figure 7. Manual odontometry

2.4.2. Automated Digital Odontometry and Odontotomy: Digital methods allow obtaining data from a series of measurements regarding the whole tooth crown. This is achieved by sectioning 3D images by parallel equally spaced sections to generate contours serving for further odontometric studies. Measuring software allows obtaining of odontometric data automatically immediately after setting of the sectioning direction. Data is collected after corresponding verification and manual correction of measurement errors.

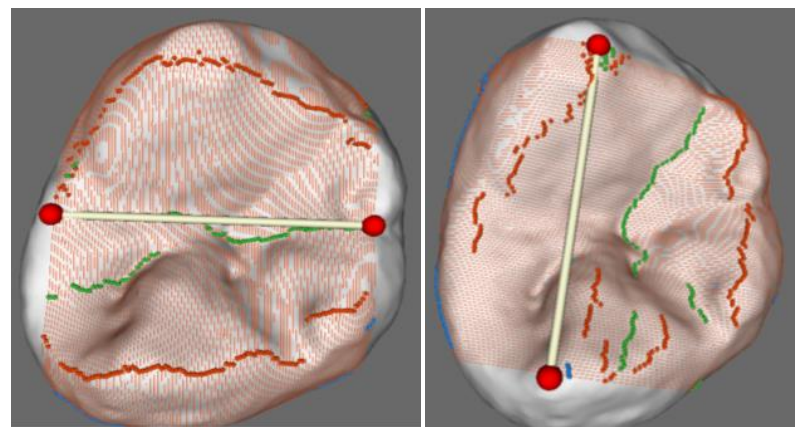

Figure 8. Two directions of odontotomy
Odontotomy, as a tooth sectioning technique, was conducted in different versions. For the comparative study of manual and automated digital methods 100 -section odontotomy in mesiodistal and vestibular-oral directions was used (Figure 8)
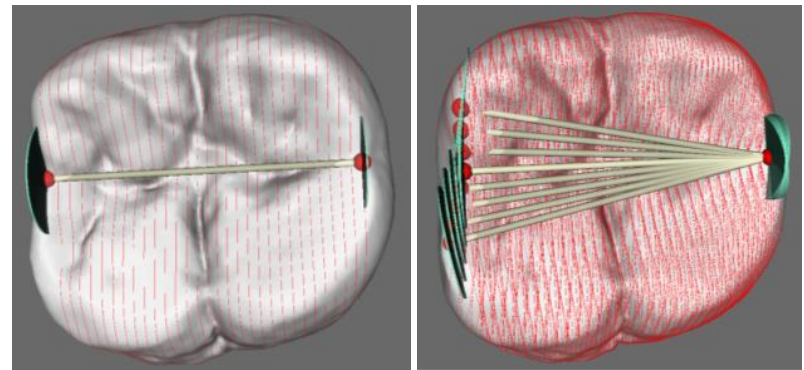

Figure 9. Varieties of odontotomic directions.

Other types of odontotomy were used for studies focused on automated digital measurement method evaluation. For estimating the influence of section direction on odontometric parameters, odontotomic series between one stable point on one side of the tooth crown and a series of movable - on the other were obtained. Movable points were set with $0.5 \mathrm{~mm}$ intervals simulating deviations caused by subjective choice of odontotomy launching landmarks; 30-section odontotomy was conducted at every direction (Figure 9).

The influence of analysed section number (can be referred to inter-section interval as well), on the measured through automated digital odontometry parameters was studied as well. Two approaches to diminution of analysed section quantity were applied. First we picked smaller selections out of maximal - 100-section odontotomy. These selections varied by section number and starting points. For instance, taking data for 20section series having equal inter-section intervals, we choose different starting points as well: zero, first, second and third sections respectively. At the other approach varying number of sectioning planes was achieved by actual constructing of various number of sections between two fixed points on studied teeth (Figure 10). From 10 to 100 -section series with steps of 10 sections were obtained. Inter-section intervals, depending on the studied tooth and sectioning rate, are in the limits of $0.06-0.88$ $\mathrm{mm}$.

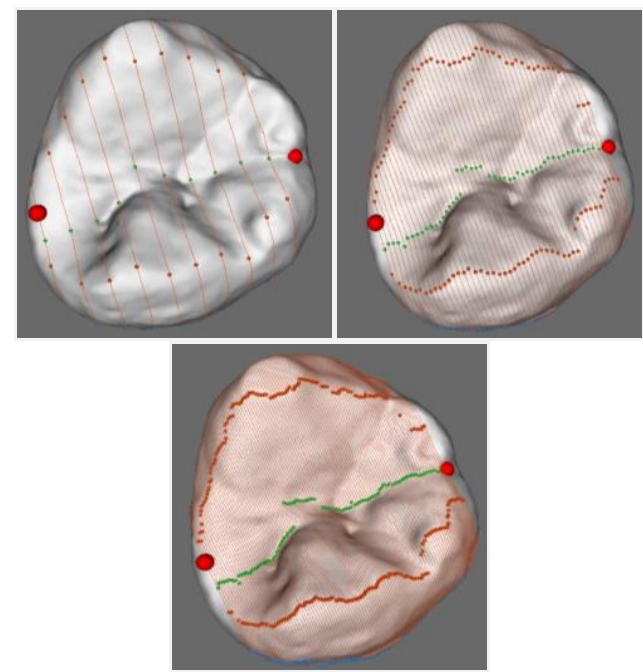

Figure 10. Progressive stages of single direction odontotomy 


\subsection{Analysis of odontometric data}

Mesio-distal and vestibular-lingual diameters (i.e. maximal sizes in these dimensions) of 10 teeth crowns were measured manually and by digital automated method. Some of parameters were chosen for re-measuring to verify the accuracy of the obtained results. As it has been the first experience of application of two above-mentioned methods to measurements of anthropological samples, we were interested in comparing of the estimated parameters of tooth crowns. The obtained results, manual and digital, were compared with each other in two separate series - mesio-distal (MD) and vestibular-lingual (VL) - calculating linear regression (1) and mean approximation error (2):

$$
\begin{aligned}
& \hat{y}=a x+b \\
& \overline{\mathrm{A}}=\frac{1}{n} \sum\left|\frac{y_{i}-\hat{y}_{i}}{y_{i}}\right| \cdot 100 \%
\end{aligned}
$$

Unlike the comparative study, for analysing digital automated odontometry method the parameter of vestibular-oral dimension of anatomical occlusal surface was chosen, which is not usually measured in classical odontometry but has been of particular interest in our previous studies. This parameter was compared at various odontotomic sectioning directions and sectioning rates.

The influence of sectioning direction on the measurement result was studied on diagrams and by means of correlation-regression analysis. Odontometric parameters obtained from every chosen direction of sections were compared to the initial, chosen as reference, direction.

In the other part of studies 100-section odontotomy was chosen as reference. Then among the 100 -section series smaller data series with equal intervals were chosen. The other approach implies similar comparisons related to data obtained through various: $10-, 20-, 30-\ldots 90$-section series, having the same direction and 100-section odontotomy as the reference. Taking into consideration the higher potential of automated digital method in obtaining various odontometric data compared to traditional methods, two types of parameters were analysed. One part - similarly to traditional methods - maximal, other average, referring to the whole occlusal surface of the studied tooth, calculated as average for vestibular-oral dimension of anatomical occlusal surface on all sections and for each section series.

\section{STUDY RESULTS}

\subsection{Comparison of parameters obtained through manual measurements and digital automated odontometry}

The results of measurements of mesio-distal and vestibularlingual diameters of 10 teeth conducted manually and via digital automated odontometry are presented in Table 11. Dental formula is presented in two, accepted in anthropology and dentistry, versions; burial numbers are indicated as well.

\begin{tabular}{|l|c|c|c|c|}
\hline \multirow{2}{*}{ Tooth } & \multicolumn{2}{|c|}{ MD, mm } & \multicolumn{2}{c|}{ VL, mm } \\
\cline { 2 - 5 } & manu & d/auto & manu & d/auto \\
\hline $\mathrm{M}_{1} \mathrm{~s}(3.6)$, Bur 1 & 10.9 & 10.980 & 10.6 & 10.734 \\
\hline $\mathrm{P}_{1} \mathrm{~s}(3.5)$, Bur 1 & 7.1 & 7.245 & 8.1 & 7.968 \\
\hline $\mathrm{M}^{3} \mathrm{~s}(2.8)$, Bur 1 & 8.8 & 8.827 & 10.6 & 10.419 \\
\hline $\mathrm{M}^{3} \mathrm{~d}(1.8)$, Bur 1 & 7.8 & 7.912 & 9.7 & 9.660 \\
\hline $\mathrm{M}^{3} \mathrm{~s}(2.8)$, Bur 3 & 8.8 & 9.474 & 10.3 & 9.236 \\
\hline $\mathrm{M}_{3} \mathrm{~s}(3.8)$, Bur 3 & 8.9 & 9.005 & 8.1 & 8.220 \\
\hline $\mathrm{M}^{2} \mathrm{~s}(2.7)$, Bur 3 & 9.5 & 9.522 & 10.9 & 10.607 \\
\hline $\mathrm{M}_{2} \mathrm{~s}(3.7)$, Bur 3 & 10.3 & 10.703 & 9.9 & 10.225 \\
\hline $\mathrm{M}_{2} \mathrm{~d}(4.7)$, Bur 3 & 10.7 & 10.363 & 10.2 & 9.999 \\
\hline $\mathrm{M}^{2} \mathrm{~s}(2.7)$, Bur 3 & 9.6 & 9.668 & 11.6 & 11.647 \\
\hline
\end{tabular}

Table 11. MD and VL odontometry; measurement results.

Comparison of measurement results by defining the linear regression for MD and VL measurements are shown on Figure 12. The mean approximation error calculated for MD series of measurements is approximately $1.7125 \%$; for VL series $2.5892 \%$.

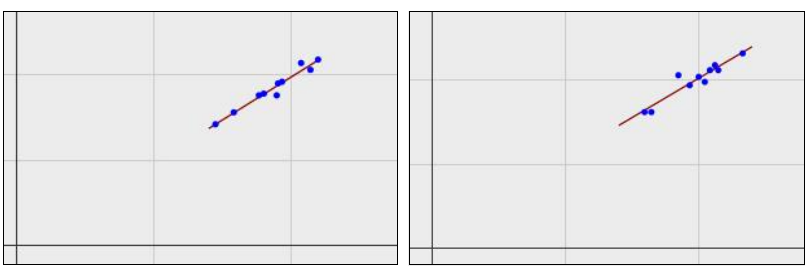

Figure 12. Linear regression graph for MD (left) and VL (right) measurements.

\subsection{Analysis of Digital Automated Odontometry Method}

3.2.1. Directions of Sectioning Planes: Changes in direction of sectioning planes affect the odontometric parameters. This tendency can be seen on diagrams of two adjacent sections comparisons revealing values of measured parameters on 30section odontotomy (Figure 13).

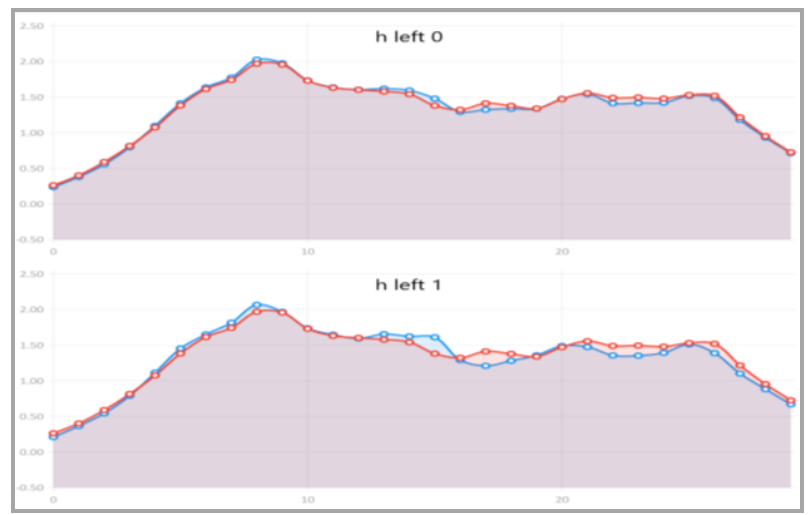

Figure 13. Diagrams of odontometric parameters at the initial sectioning direction (red line) and two deviations: adjacent to it (upper blue line) and next one (lower blue line) 
Calculations show that differences in average odontometric parameters gradually (in some cases - shaprly - depending on the studied sample) grow with the increase of deviation of section defining axis from the initial direction.

3.2.2. Number of Analysed Sections in 100-Section Series: Reduction in number of analysed sections reveals variability of average parameters. Some cases demonstrate closest data to 100-section odontotomy on 50-section series (Figure 14).

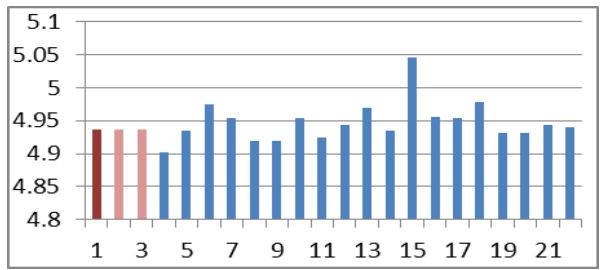

Figure 14. Average odontometric parameters: 100-section series (red), 50-section series (pink)

Other studied samples have maximal similarity on 80 - or 20 section series. They can also reveal the influence of starting section choice on the obtained data, i.e. 20-section odontotomy data differs if we start at 0,1 or 2 sections (Figure 15).

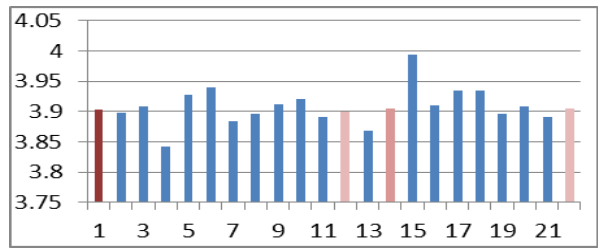

Figure 15. Average odontometric parameters: 100 -section series (red), 20-section series, start at 2nd section (dark pink); 20section series, start at 0 section (pink, left) and 80-section series (pink, right).

3.2.3. Number of Sections at Various Rate Series: The general tendency shows a slight increase in average odontometric parameters with initial reduction of section quantity. Further reduction brings to decrease (gradual or sharp) of average parameters. We link this pattern of changes with initial reduction of data from marginal sections (they are few in quantities but are the lowest in their values). Further reduction of maximal parameter frequencies leads to decrease of average parameters (Figure 16).

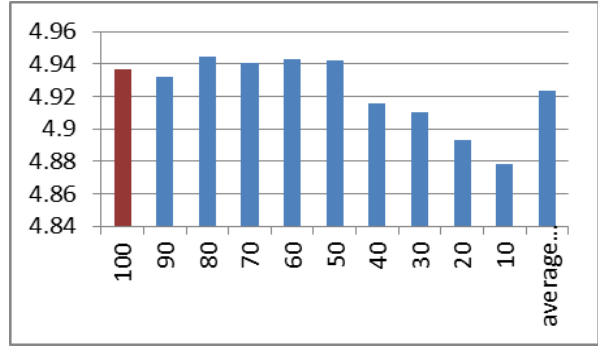

Figure 16. Average odontometric parameters at different section rates.

The other part of the current study referred to the maximal odontometric parameters. As could be expected, increase of section quantity would bring to precise locating of the required position of maximal dimension (Figure 17). Nevertheless in some cases higher values of maximal parameters can be observed at section rates lower than maximal; 90-, 80-, and 70section odontotomy can be found to be more effective in positioning of the required section than 100-section odontotomy (Figures 17, 18). Maximal parameters vary differently from tooth to tooth but have a general declining trend at minimal section rates (10-section odontotomy).

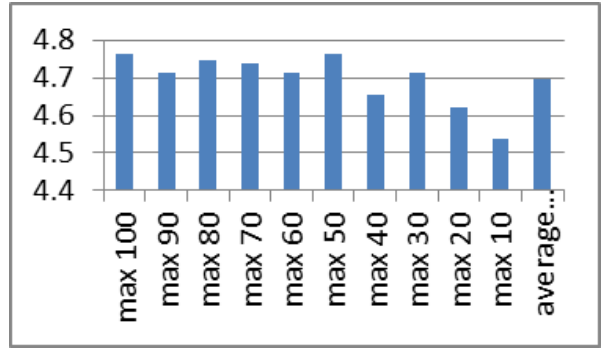

Figure 17. Maximal odontometric parameters at different various rates on a premolar.

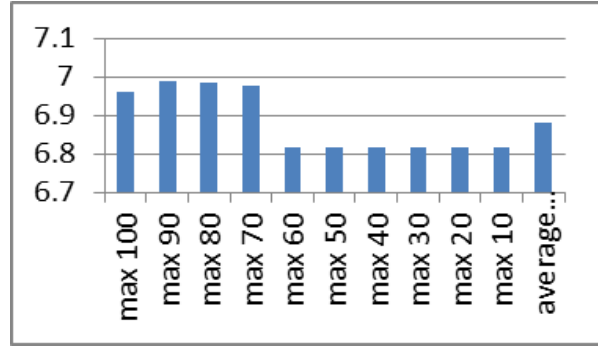

Figure 18. Maximal odontometric parameters at vatious section rates on a molar.

\section{CONCLUSIONS}

This study related to various matters of odontometry, manual and digital, is dealing with comparative studies and analysis of measuring methods.

Our study has showed certain differences between results obtained by application of manual measurement methods widely accepted in anthropology and automated digital odontometry. In view of the fact that the same teeth (and their 3D images) were measured though two methods it would have been difficult to expect obtaining widely divergent parameters. This can be seen on the results of calculation of mean approximation error, thus both methods can be applied for odontmetry. The same calculations reveal differences in MD and VL measurements. Mesio-distal (MD) direction of measurements provides more uniform results in comparison to vestibulo-lingual (VL). Here we can see relations with the choice of measurements' direction, which is sometimes a challenge during manual odontometry, especially on upper molars (half of our studied teeth are upper molars). In fact, choosing of grooves and cement-enamel junction as orientation landmarks for manual odontometry direction detection causes deviations from vestibular-oral dimension. Digital methods are aimed to be adhered to more objective choice of measurements' directions. In this regard other parts of the study presented in the article were carried out. Odontotomy, forming insofar the initial data for further measurements, remains the most subjective part of automated 
digital method. Thus a significant part of the study is focused on analysis of sectioning direction and rate influence on the obtained parameters. Even seemingly minor changes in sectioning directions cause noticeable changes to odontometric data. This changes, smooth or abrupt, which depends on tooth morphology and on the observed parameter, are proportional to the degree of sectioning direction deviation.

Odontotomy has proved to be a method improving analytical capabilities of odontometric methods. This can be seen while studying parameters, maximal and average, which is inaccessible by application of manual methods, at various sectioning rates. In accordance to the results of our study a special attention should be paid to further development of automated odontometric software in the direction of maximal parameter area detection, choice of odontotomy launching landmarks and others.

In order to provide for more profound studies of tooth shape it is necessary to analyse parameters obtained from contours as well as tooth shape features which related to their 3D structure. Accumulated data forms the basis for morphological tooth shape description through measurements. Currently automated digital odontometry combining 3D shape analysis including principal component analysis and tooth contour analysis suggest obtaining results of demand in dental, anthropological and archaeological studies.

\section{ACKNOWLEDGEMENTS}

The work was performed with the support by Grant 17-2904509 of Russian Foundation for Basic Research (RFBR).

We would like to thank colleagues from Digital Dentistry Associations of Russia and Armenia for their support in scanning.

\section{REFERENCES}

Gaboutchian A.V., Knyaz V.A., Bolshakov G.V., 2017 a. Odontometrics And Occlusiometric Research On Computer Three-Dimensional Images Of The Premolars And Molars. Vestnik Antropologii, № 3 (39), pp. 98-115.

Gaboutchian A.V., Knyaz V.A., Leybova N.A., 2017 b. Measurements Of Cuspal Slope Inclination Angles In Palaeoanthropological Applications. The International Archives of the Photogrammetry, Remote Sensing and Spatial Information Sciences, Volume XLII-2/W4, 2017 2nd International ISPRS Workshop on PSBB, 15-17 May 2017, Moscow, Russia, doi:10.5194/isprs-archives-XLII-2-W41852017.

Fleming P.S., Marinho V., Johal A., 2011. Orthodontic Measurements On Digital Study Models Compared With Plaster Models: A Systematic Review. Orthodont Craniofac Res; 14, 1 16. doi: 10.1111/j.1601-6343.2010.01503.x

Hasegawa Y, Amarsaikhan B., Chinvipas N., Tsukada Sh.-I., Terada K., Uzuka S., Miyashita W., Iguchi S., Arai K., Kageyama I., Nakahara S., 2014. Comparison of mesiodistal tooth crown diameters and arch dimensions between modern Mongolians and Japanese. Odontology, 102, pp. 167-175 doi: 10.1007/s10266-013-0130-5
Hillson S., FitzGerald C., Flinn H., 2005. Alternative Dental Measurements:Proposals and Relationships with Other Measurements. American Journal of Physical Anthropology, 126, pp. 413-426.

Horton H.M.I., Miller J.R., Gaillard P.R., Larson B.E., 2010. Technique Comparison for Efficient Orthodontic Tooth Measurements Using Digital Models. Angle Orthodontist, 2010, Vol 80, No 2, pp 254-261.

Irish J.D., Scott G.R., 2016. A Companion to Dental Anthropology. John Wiley \& Sons, Inc.

Knyaz V.A., Gaboutchian A.V., 2016. Photogrammetry-based Automated Measurements for Tooth Shape and Occlusion Analysis // The International Archives of the Photogrammetry, Remote Sensing and Spatial Information Sciences, Volume XLI-B5, doi:10.5194/sprsarchives-XLI-B5-849-2016.

Paredes, V., Tarazona, B., Zamora, N., Hernandez, Y., San José, V., 2015. A Comparative Study of Reproducibility, Reliability and Accuracy in Measuring Mesiodistal Tooth Size Using Four Different Methods - 2D Digital, 3D CBCT, 3D Segmented and 3D Intraoral Scanner Method. Issues Contemporary Orthodontics. http://dx.doi.org/10.5772/59366.

Peiris R., Satake T., Kanazawa E., 2013. Fluctuating asymmetry of the permanent mandibular molars in a Japanese population. Odontology, 101, pp. 15-21. doi: 10.1007/s10266-012-0064-3

Scott G.R., Turner Ch.G., 1997. The Anthropology of Modern Human Teeth. Cambridge University Press.

Sekikawa M., Kanazawa E., Ozaki T., Brown T., 1988. Principal Components Analysis of Intercusp Distances on the Lower First Molars of Three Human Populations. Archives of Oral Biology, 33, pp. 535-541.

Townsend, G., Kanazawa, E., Tokuyama, H., 2012. New Directions in Dental Anthropology: paradigms, methodologies and outcomes. University of Adelaide Press. 